\title{
Stem cell self-renewal versus differentiation: Tumor suppressor Mei-P26 and miRNAs control the balance
}

\author{
Run Shen ${ }^{1}$, Ting Xie ${ }^{1}$ \\ ${ }^{I}$ Stowers Institute for Medical Research, 1000 East $50^{\text {th }}$ Street, Kansas City, MO 64110, USA \\ Correspondence:RUS@stowers-institute.org \\ Cell Research (2008) 18:713-715. doi: 10.1038/cr.2008.79; published online 3 July 2008
}

Stem cells, which can self-renew and produce different cell types, have been shown to be regulated by extrinsic signals and intrinsic factors. Drosophila ovarian germline stem cells (GSCs), representing one of the well-studied stem cells, continuously proliferate and generate differentiated cystoblasts, which further develop into oocytes. In the Drosophila ovary, cap cells form the GSC niche, which produces the BMP signal for maintaining GSC self-renewal [1]. BMP signaling directly represses the transcription of bam, a key differentiation factor, to prevent GSC differentiation and thereby maintain self-renewal. Bam acts with its partner Bgen to sufficiently drive GSC differentiation. Disruption of BMP signaling leads to GSC premature differentiation and loss, while elimination of Bam/Bgcn function results in accumulation of GSC-like cells. mei-P26, which was initially identified for its role in meiotic recombination, has also been shown to be required for GSC daughter differentiation since its mutant ovaries contain more GSC-like cells [2]. As expected, mei-P26 and bam genetically interact with each other to regulate germ cell differentiation. mei-P26 encodes a protein containing a RING finger B-box Coiled-Coil (RBCC) and a NHL (NCL-1, $H \mathrm{~T} 2 \mathrm{~A}$, and Lin-41) domain [2]. Recently, the miRNA pathway is also shown to be required for controlling GSC self-renewal since mutations in Dicer-1, Agol, and loquacious, which are involved in miRNA production and function in Drosophila, cause rapid loss of GSCs [3-5]. Small 23-25 nt long miRNAs can regulate gene expression through translation repression and mRNA degradation by binding to the 3' untranslated region (UTR) of their target mRNAs [6]. Interestingly, the miRNA pathway controls GSC self-renewal not by repressing bam [3-5]. However, it remains unclear how mei-P26 and bam, suppressors of GSC-like tumors, negatively interact with the miRNA pathway to control the balance between self-renewal and differentiation. In a recent issue of Nature, Neumuller et al. have provided the missing link between mei-P26/bam-medatiated differentiation pathway and the self-renewal-promoting miRNA pathway [7].

Neumuller et al. have provided three lines of evidence supporting the idea that mei-p26 works closely with bam to control germ cell differentiation [7]. First, Mei-p26 expression is developmentally delayed in comparison with Bam, suggesting that Mei-P26 functions after Bam in the regulation of germ cell differentiation. Second, mei-P26 is genetically epistatic to bam. Normally, bam overexpression is sufficient to induce GSC differentiation, but it fails to induce mei-P26 mutant GSCs to differentiate, indicating that bam can not function without mei-P26. Third, mei$P 26$ overexpression fails to rescue the bam mutant GSC-like tumor phenotype, indicating that mei-P26 function in the regulation of germ cell differentiation is also dependent on bam. These results are consistent with the previous finding that a mutation in bam can enhance germ cell differentiation defects of meiP26 mutants [2]. However, it remains unclear how Bam and Mei-P26 regulate each other in germ cell differentiation.

Neumuller et al. then provided mechanistic insight into how the bam/ mei-P26-directed differentiation axis affects the GSC self-renewal process [7]. Interestingly, affinity purification and co-IP experiments show that MeiP26, as well as its paralog, Brat (brain tumor), can interact with Agol through the NHL domain. In addition, the NHL domain is required for Mei-P26 function in germ cell differentiation. Although the RISC complex is involved in the regulation of miRNA-mediated translation repression or mRNA degradation [6], this study suggests that the Agocontaining RISC complex also has a role in the regulation of miRNA expression. In mei-P26 mutant ovaries, expression levels of some miRNAs increase, while overexpression of mei-P26 in bam mutants can decrease miRNA expression. bantam, which is known to promote proliferation and inhibit apoptosis in the Drosophila imaginal disc [8], can promote GSC self-renewal since its heterozygous mutant ovaries have reduced GSC number. Its expression and activity is upregulated in mei-P26 mutant germ cells to sustain continuous germ 
cell proliferation. Finally, a mutation in loqs can also suppress the sterility of mei-P26 mutant female, indicating that Mei-P26 promotes germ cell differentiation by antagonizing the selfrenewal-promoting miRNA pathway. These results show that Mei-P26 can interact with Agol to inhibit miRNA expression and function during germ cell differentiation, providing important insight into how Mei-P26 controls germ cell differentiation.

Although it remains unclear how the miRNA pathway controls GSC selfrenewal, this study on Mei-P26 has offered some insight into how the miRNA pathway and Bam/Mei-P26 regulate the balance between self-renewal and differentiation [7]. Brat is known to interact with a Pum/Nos complex through its NHL domain and function as a translational repressor [9]. Interestingly, Pum and Nos are required for controlling GSC self-renewal by preventing differentiation $[10,11]$. In C. elegans, a pum homolog, puf-9, and let-7 miRNA directly bind to the $3^{\prime} \mathrm{UTR}$ of hunchback mRNA to suppress its expression [12]. Since Agol along with miRNAs binds to 3'UTRs of target mRNAs to repress translation or trigger mRNA degradation, it raises an interesting possibility that the target mRNAs, which encode protein products important for germ cell differentiation, may contain Pum/Nos binding sites and miRNA binding sites at their 3'UTRs, which bring together the Pum/Nos complex and the Ago1containing RISC complex. In order to trigger germ cell differentiation, MeiP26 can interact with Agol to antagonize the miRNA/Pum/Nos-mediated translation repression and thus turn on expression of germ cell differentiationpromoting proteins. This model can explain how the miRNA pathway and the Pum/Nos complex promote GSC self-renewal, and how Mei-P26/Bam inhibit GSC self-renewal and thus promote differentiation (Figure 1).

Since Mei-P26 and Brat, two NHLcontaining proteins, function as tumor

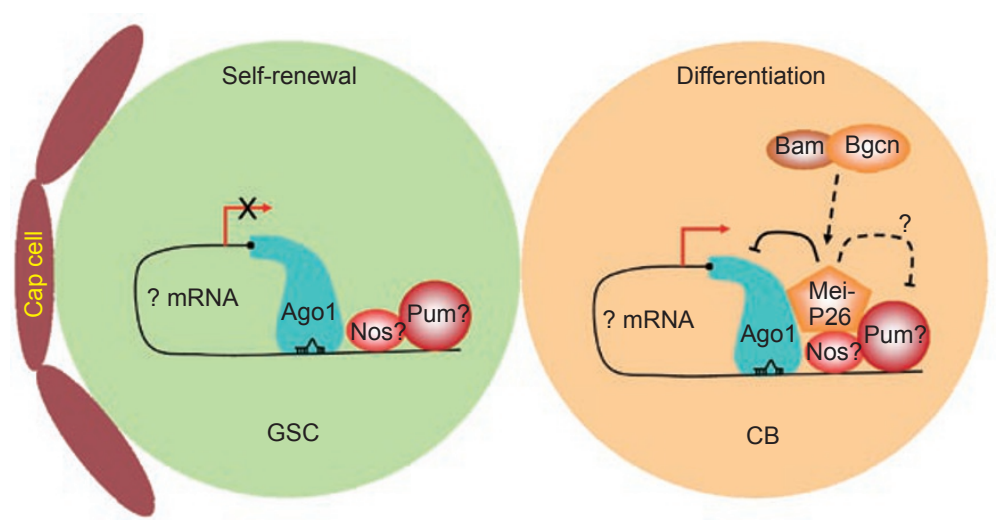

Figure 1 miRNA/nos/pum self-renewal pathway and bam/mei- $P 26$ differentiation pathway control the balance between GSC self-renewal and differentiation. nos and pum maintain GSC self-renewal by preventing differentiation. mei-P26 works with bam/bgcn to promote cystoblast (CB) differentiation by interacting with Ago1, or possibly Nos and Pum to turn on target gene expression.

suppressors by interacting with Ago 1 to repress stem cell activities in the Drosophila ovary and the brain, respectively, this study by Neumuller et al. sheds new light on how tumor suppressors and miRNAs control stem cell function and cell proliferation at the molecular and cellular level [7]. This study has also provided significant insight into how miRNA-mediated translational repression can be relieved and the translation process resumes. However, it remains unclear how Mei-P26 can mechanistically inactivate miRNA-mediated translational repression through interacting with Ago1. Further revelation of the detailed mechanism underlying the antagonizing functions between MeiP26 and miRNAs will surely boost our understanding of the miRNA pathway in tumorigenesis, and lead to possible new cancer treatments.

\section{References}

1 Kirilly D, Xie T. The Drosophila ovary: an active stem cell community. Cell Res 2007; 17:15-25.

2 Page SL, McKim KS, Deneen B, Van Hook TL, Hawley RS. Genetic studies of mei-P26 reveal a link between the processes that control germ cell proliferation in both sexes and those that control meiotic exchange in Drosophila.
Genetics 2000; 155:1757-1772.

3 Yang L, Chen D, Duan R, et al. Argonaute 1 regulates the fate of germline stem cells in Drosophila. Development 2007; 134:4265-4272.

4 Park JK, Liu X, Strauss TJ, McKearin DM, Liu Q. The miRNA pathway intrinsically controls self-renewal of drosophila germline stem cells. Curr Biol 2007; 17:533-538.

5 Jin Z, Xie T. Der-1 maintains Drosophila ovarian stem cells. Curr Biol 2007; 17:539-544.

6 Valencia-Sanchez MA, Liu J, Hannon GJ, Parker R. Control of translation and mRNA degradation by miRNAs and siRNAs. Genes Dev 2006; 20:515524.

7 Neumuller RA, Betschinger J, Fischer A, et al. Mei-P26 regulates micro RNAs and cell growth in the Drosophila ovarian stem cell lineage. Nature 2008; in press.

8 Brennecke J, Hipfner DR, Stark A, Russell RB, Cohen SM. bantam encodes a developmentally regulated microRNA that controls cell proliferation and regulates the proapoptotic gene hid in Drosophila. Cell 2003; 113:25-36.

9 Sonoda J, Wharton RP. Drosophila Brain Tumor is a translational repressor. Genes Dev 2001; 15:762-773.

10 Forbes A, Lehmann R. Nanos and Pumilio have critical roles in the development and function of Drosophila germline stem cells. Development 1998; 125:679-690. 
11 Wang Z, Lin H. Nanos maintains germline stem cell self-renewal by preventing differentiation. Science 2004; 303:20162019.
12 Nolde MJ, Saka N, Reinert KL, Slack FJ. The Caenorhabditis elegans pumilio homolog, puf-9, is required for the
3'UTR-mediated repression of the let-7 microRNA target gene, hbl-1. Dev Biol 2007; 305:551-563. 